\title{
Integrated nanoelectronic-photonic devices and bioresorbable materials
}

\author{
John A. Rogers $(\bowtie)$ \\ Departments of Materials Science and Engineering, Biomedical Engineering and Neurological Surgery, Simpson Querrey Institute for Bioelectronics, \\ Northwestern University, Evanston, IL 60208, USA
}

(c) Tsinghua University Press and Springer-Verlag GmbH Germany, part of Springer Nature 2021

I am honored receive, with Prof. Zhao, this year's Nano Research Award. In my case, the recognition is largely an acknowledgement of the scientific advances achieved by a diverse set of graduate students, undergraduates and postdoctoral fellows as they passed through my group over the years-talented, dedicated, creative, collaborative people, of the very highest caliber. Several of these former group members, each now leading large and highly successful academic research programs of their own, contributed outstanding articles to this special issue-21 in total! I would like to extend my special thanks to them-I feel a strong sense of pride and joy in their many impressive accomplishments as pioneering, independent researchers. The topics of these articles span a wide range, as a powerful set of projects at the forefront of nanoscience and nanotechnology, briefly summarized by topic area in the following.

Many of the papers feature the role of light and optics in advanced nanosystems. A comprehensive review article by Prof. Cunjiang Yu (a former postdoctoral fellow) and his students at the University of Houston summarizes the stateof-the-art in flexible photovoltaic devices as power sources for skin-mounted electronic devices [1]; as a complement to this piece on interactions with ambient light, Prof. Young Min Song (a former postdoctoral fellow) and his group at Gwanju Institute of Science and Technology (GIST) describe bio-integrated platforms that use light to probe body processes [2]; Prof. Xing Sheng (a former postdoctoral fellow) writes with his students at Tsinghua University on monolithically integrated microscale components that emit and detect light using photon recycling concepts with unique capabilities in characterizing soft biological tissues, such as the skin [3]; generation and detection of light using flexible/stretchable platforms are important in this broader context, as highlighted in an article by Prof. Dae-Hyeong Kim (a former PhD student and postdoctoral fellow) and his group at Seoul National University [4]; as the basis for sophisticated methods for electronic sensing of light, Prof. Sung Hun Jin (a former postdoctoral fellow) and his students at Incheon National University describe the latest progress in two-dimensional (2D) nanomaterials for converting light to frequency modulated electronic signals [5] and, in a separate article, recent work on high performance transistors for controlling microscale lightemitting diodes (LEDs) and other components [6]; Prof. Seokwoo Jeon (one of our first PhD students) and his group at the Korean Advanced Institute for Science and Technology (KAIST) write about powerful approaches that exploit light to fabricate complex nanostructures, including those at large-scales and in threedimensional (3D) configurations [7]; in terms of unusual optical nanomaterials by chemical synthesis, Prof. Yugang Sun (a former postdoctoral fellow) and his students at Temple University describe methods for forming silica layers on titania nanoparticles to control their plasmonic properties through nitridation [8]; in work that shares some common chemistry concepts, Prof. Yujie Xiong (a former postdoctoral fellow) at University of Science and Technology of China (USTC) describes in an article co-authored with his students the use of carbon nanomaterials as catalysts in nitrogen reduction reactions [9].

Another set of papers highlights nanomaterials and their roles in unique classes of electronic devices and biosensors. As an illustration in biochemical sensing, Prof. Xian Huang (a former postdoctoral fellow) and his co-authors at Tianjin University, report the use of metal-organic frameworks in unusual types of implantable devices [10]; silicon nanomembranes represent another important category of nanomaterial, with interesting fundamental properties and wide-ranging applications in flexible, stretchable and transient electronics, as highlighted in an article by Prof. Jong-Hyun Ahn (a former postdoctoral fellow) and his students at Yonsei University [11]; graphene remains a topic of high interest in the nanoscience community, and Prof. Zheng Yan (a former postdoctoral fellow) and his group report on the latest advances in laser induced graphene on polymeric substrates and their applications [12]; as another form of $\mathrm{sp}^{2}$ hybridized carbon, Prof. Qing Cao (a former PhD student) contributes an article on the latest work on arrays of carbon nanotubes as a semiconductor material for high performance transistors and integrated circuits [13].

An additional frontier area is in nanotechnology for interfaces to living systems, sometimes using design principles inspired by biology. Prof. Jang-Ung Park (a former PhD student) and his group at Yonsei University write about the broad range of microsystems technologies that are capable of integrating on or in the brain, for monitoring and stimulation via physical and electrophysiological mechanisms [14]; in related directions, Prof. Hui Fang (a former postdoctoral fellow) reports on work with his students at Northeastern University (recently moved to Dartmouth University) on circuit level strategies to minimize electrical artifacts in neural recordings captured with dense, ultrathin electrodes of conducting polymers [15]; in an expanded applications focus, Prof. Ki Jun Yu (a former PhD student) also at Yonsei University, co-authors an article with this 
students on carbon-nanomaterials for pressure sensors in health monitoring systems [16]; in a contribution from the University of California at San Diego, Prof. Sheng Xu (a former postdoctoral fellow) describes work from his group on fabric-mounted wireless devices for electrophysiological sensing with performance enhanced by interfacial nanoparticles of materials with high dielectric constants [17]; on additional materials work in this broader context with his students at USTC, Prof. Hangxun Xu (a former postdoctoral fellow) writes about conducting polymers and nanostructures formed by them as the basis for soft electronics, with potential in bio-interfaced systems [18]; an article from Prof. Tae-il Kim's (a former postdoctoral fellow) group at Sungkyunkwan University describes an interesting concept in which porous elastomers and boron nitride nanomaterials can be used to engineer thermal transport properties for improved performance of stretchable electronic devices [19]; from the standpoint of circuit and component designs, Prof. Keon Jae Lee (one of our earliest PhD students) and his students at KAIST write about neuromorphic components and circuit designs [20]; and finally, as manufacturing science is important in all of the systems described here, Prof. Heung Cho Ko (a former postdoctoral fellow) contributes an article from his group at GIST on clever ways to use micro/nanostructured surfaces as the basis for control of adhesion in schemes for manipulating nanomaterials for device integration via methods of transfer printing [21].

For more than twenty years, my group has been active in these and other areas of research that involve nanomaterials and nanotechnologies-where the former students and postdoctoral fellows mentioned above each made major contributions while part of my laboratory. His current efforts can be organized into six different categories. The first is in biodegradable materials for transient electronics, defined by systems that support a well-defined operational lifetime followed by a period during which the hardware gradually disappears. One class of this technology is designed to dissolve in a controlled manner to eco/biocompatible end products when exposed to water. Complete sets of active and passive materials serve as an enabling basis for these systems, where nanostructured forms of monocrystalline silicon-wires, membranes and ribbons-represent the material of choice for the semiconductor. The approach exploits a previously underappreciated fact in solid state materials chemistry-device-grade silicon dissolves by hydrolysis to silicic acid and hydrogen when immersed in water at physiological temperatures and $\mathrm{pH}$, with rates of a few nanometers per day. Nanostructured forms of silicon, sufficiently thick to support high performance operation in transistors, diodes, photodetectors and other essential electronic components, offer dimensions sufficiently small to dissolve completely in biofluids on timescales of days to weeks. When combined with water-soluble dielectrics and conductors, this nanoscale chemistry serves as the foundation for advanced silicon-based temporary biomedical implants, with applications in bioelectronic stimulators, sensors, drug release vehicles and other engineered systems that support an important function (diagnostic, therapeutic) during the course of a transient biological process (e.g., wound healing, tissue growth, etc.) and then vanish completely and naturally from the body, to avoid the need for surgical extraction.

A second area of our research is in hard/soft composite materials as enabling constructs for stretchable electronics. Here, high-modulus inorganic nanomaterials combine in optimized ways with low-modulus elastomers to yield hybrid classes of materials that offer the favorable electronic properties of the former with the soft mechanics of the latter. The results create routes to unusual electronic devices, with an ability to naturally and gently integrate onto the surfaces of soft, time dynamic biological tissues-from skin-like wearables for monitoring physiological health, to high-resolution sheets for mapping brain activity. Both transient and stretchable electronics rely critically on methods that enable heterogeneous integration of highly dissimilar materials, including those in nanostructured forms. Development of nanomanufacturing techniques for building such structures represents a third area of research. The most successful schemes exploit elastomeric "stamps" and kinetic control over adhesion in viscoelastic materials as means to manipulate micro/nanostructured materials into monolithic, multilayer assemblies. Taken together with methods for epitaxial growth and liftoff of nanomaterials from wafer hosts, these methods can be used to construct wide varieties of heterogeneously integrated semiconductor devices-not only transient and stretchable electronics, but collections of microscale light emitting diodes for information displays with unmatched performance and arrays of multi-junction, microconcentrator solar cells for photovoltaic modules with world record efficiencies.

Exploratory application possibilities that follow from these materials and assembly approaches represent the fourth and fifth areas of our research. The first of these is in materials approaches to bio-inspired systems. Here, the emphasis is on architectures, growth strategies and integration techniques that create opportunities to leverage designs for electronic/ optoelectronic systems that take inspiration from the biological world. Notable outcomes are in digital imaging platforms with layouts in the hemispherical geometries of the human and arthropod eye, in adaptive display devices that reproduce essential features of cephalopod skin and in electronic microfliers with layouts of wind-dispersed seeds. The second application area is in cellular-scale, injectable optoelectronic systems that allow interconnected collections of active light emitters, photodetectors, electrical stimulators and other components to be deployed directly into the deep brain and into various areas of the peripheral nervous system, for use as tools in neuroscience and as advanced bioelectronic medicines.

An outgrowth of these activities in neural interfaces forms our sixth area of research. Here, the work addresses nanomaterials-based routes to extended, 3D mesoscale frameworks that support multi-modal, volumetric interfaces to soft, living tissues. Versatile schemes exploit deterministic processes in non-linear buckling mechanics to induce controlled, geometric transformation of two-dimensional micro/ nanostructures into complex, well-designed, 3D architectures that incorporate diverse materials and devices. The results yield broad possibilities in active microsystems technologies with 3D designs, from advanced microelectromechanical systems, to templates for materials growth, to interfaces to small-scale tissue constructs such as organoids and assembloids.

These efforts, along with those of many other groups in nanoscience/nanotechnology, suggest that robust streams of creative ideas, powerful techniques and clever design approaches will continue to create vibrancy and excitement in this field. An important trend, most notable in recent years, is an expansion of research interests beyond techniques for nanomaterials growth and synthesis, to essential aspects of device engineering and manufacturing. The field is quickly evolving beyond basic studies that address the question "How can one synthesize nanomaterials in a controlled way to achieve desired properties?" to include programs in engineering science that aspire to respond to the challenge of "How does one exploit these nanomaterials to realize systems with useful functions 
that cannot be achieved using conventional approaches?" This broadening into a more comprehensive and ambitious portfolio of programs represents a source of optimism for our collective ability to translate fundamental academic advances in nanoscience into nanotechnologies that can be benefit society. Many of the projects in my group focus on human health, but additional opportunities are in cost efficient methods for purification of water, in emerging systems for virtual/augmented reality and remote learning, and in technologies to meet growing needs in energy generation and storage. As a community, we are poised to make important contributions to solving these and other grand challenges faced by humanity-we must continue to work boldly and aggressively toward these lofty goals.

\section{Reference}

[1] Kan, F.; Ershad, F.; Rao, Z.; Yu, C. J. Flexible organic solar cells for biomedical devices. Nano Res. 2021, 14, 2891-2903.

[2] Yoo, Y. J.; Heo, S.-Y.; Kim, Y. J.; Ko, J. H.; Mira, Z. F.; Song, Y. M. Functional photonic structures for external interaction with flexible/wearable devices. Nano Res. 2021, 14, 2904-2918.

[3] Ding, H.; Lv, G. Q.; Shi, Z.; Cheng, D. L.; Xie, Y.; Huang, Y. X.; Yin, L.; Yang, J.; Wang, Y. T.; Sheng, X. Optoelectronic sensing of biophysical and biochemical signals based on photon recycling of a micro-LED. Nano Res. 2021, 14, 3208-3213

[4] Song, J.-K.; Kim, M. S.; Yoo, S.; Koo, J. H.; Kim, D.-H. Materials and devices for flexible and stretchable photodetectors and lightemitting diodes. Nano Res. 2021, 14, 2919-2937.

[5] Seo, S. G.; Kim, S. Y.; Jeong, J.; Jin, S. H. Progress in light-to-frequency conversion circuits based on low dimensional semiconductors. Nano Res. 2021, 14, 2938-2964.

[6] Seo, S. G.; Jeong, J.; Kim, S. Y.; Kumar, A.; Jin, S. H. Reversible and controllable threshold voltage modulation for n-channel $\mathrm{MoS}_{2}$ and p-channel $\mathrm{MoTe}_{2}$ field-effect transistors via multiple counter doping with ODTS/poly-L-lysine charge enhancers. Nano Res. 2021, 14, 3214-3227.

[7] Nam, S.-H; Hyun, G.; Cho, D.; Han, S.; Bae, G.; Chen, H.; Kim, K.; Ham, Y.; Park, J.; Jeon, S. Fundamental principles and development of proximity-field nanopatterning toward advanced 3D nanofabrication. Nano Res. 2021, 14, 2965-2980.

[8] Wei, Q. L.; Kuhn, D. L.; Zander, Z.; DeLacy, B. G.; Dai, H.-L.; Sun, Y. G. Silica-coating-assisted nitridation of $\mathrm{TiO}_{2}$ nanoparticles and their photothermal property. Nano Res. 2021, 14, 3228-3233.

[9] Zhang, W. Q.; Mao, K. K.; Low, J. X.; Liu, H. J.; Bo, Y. N.; Ma, J.; Liu, Q. X.; Jiang, Y. W.; Yang, J. Z.; Pan, Y. et al. Working-in-tandem mechanism of multi-dopants in enhancing electrocatalytic nitrogen reduction reaction performance of carbonbased materials. Nano Res. 2021, 14, 3234-3239.

[10] Li, Y.; Ling, W.; Liu, X. Y.; Shang, X.; Zhou, P.; Chen, Z. R.; Xu, H.; Huang, X. Metal-organic frameworks as functional materials for implantable flexible biochemical sensors. Nano Res. 2021, 14, 2981-3009.

[11] Sarkar, A.; Lee, Y.; Ahn, J.-H. Si nanomebranes: Material properties and applications. Nano Res. 2021, 14, 3010-3032.

[12] Xu, Y. D.; Fei, Q. H.; Page, M.; Zhao, G. G.; Ling, Y.; Chen, D.; Yan, Z. Laser-induced graphene for bioelectronics and soft actuators. Nano Res. 2021, 14, 3033-3050.

[13] Cao, Q. Carbon nanotube transistor technology for More-Moore scaling. Nano Res. 2021, 14, 3051-3069.

[14] Kwon, Y. W.; Jun, Y. S.; Park, Y.-G.; Jang, J.; Park, J.-U. Recent advances in electronic devices for monitoring and modulation of brain. Nano Res. 2021, 14, 3070-3095.

[15] Qiang, Y.; Gu, W.; Liu, Z. H.; Liang, S. C.; Ryu, J. H.; Seo, K. J.; Liu, W. T.; Fang, H. Crosstalk in polymer microelectrode arrays. Nano Res. 2021, 14, 3240-3247.

[16] Kang, K.; Park, J.; Kim, K.; Yu, K. Y. Recent developments of emerging inorganic, metal and carbonbased nanomaterials for pressure sensors and their healthcare monitoring applications. Nano Res. 2021, 14, 3096-3111.

[17] Chen, X. J.; Gao, X. X.; Nomoto, A.; Shi, K. R.; Lin, M. Y.; Hu, H. J.; Gu, Y.; Zhu, Y. Z.; Wu, Z. H.; Chen, X. et al. Fabric-substrated capacitive biopotential sensors enhanced by dielectric nanoparticles. Nano Res. 2021, 14, 3248-3252.

[18] Wang, T.; Bao, Y. W.; Zhuang, M. D.; Li, J. C.; Chen, J. C.; Xu, H. X. Nanoscale engineering of conducting polymers for emerging applications in soft electronics. Nano Res. 2021, 14, 3112-3125.

[19] Kang, S. J.; Hong, H.; Jeong, C.; Lee, J. S.; Ryu, H.; Yang, J.; Kim, J. U.; Shin, Y. J.; Kim, T. Avoiding heating interference and guided thermal conduction in stretchable devices using thermal conductive composite islands. Nano Res. 2021, 14, 3253-3259.

[20] Sung, S. H.; Kim, T. J.; Shin, H.; Namkung, H.; Im, T. H.; Wang, H. S.; Lee, K. J. Memory-centric neuromorphic computing for unstructured data processing. Nano Res. 2021, 14, 3126-3142.

[21] Yoo, J. I.; Kim, S. H.; Ko, H. C. Stick-and-play system based on interfacial adhesion control enhanced by micro/nanostructures. Nano Res. 2021, 14, 3143-3158. 